\title{
Lysyl oxidase promotes anaplastic thyroid carcinoma cell proliferation and metastasis mediated via BMP1
}

\author{
Yujia Liu ${ }^{1}$, Yiwen Zhang ${ }^{1,2}$, Zhuo Tan ${ }^{2,3}$, Jiafeng Wang ${ }^{2,3}$, Ying Hu ${ }^{1}$, Jiao Sun ${ }^{4}$, Meihua Bao ${ }^{5}$, Ping Huang ${ }^{1,2}$, \\ Minghua $\mathrm{Ge}^{2,3}$, Young Jun Chai ${ }^{6}$, Chuanming Zheng ${ }^{2,3}$ \\ ${ }^{1}$ Clinical Pharmacy Center, Department of Pharmacy, Zhejiang Provincial People's Hospital; Affiliated People's Hospital, Hangzhou Medical \\ College, Hangzhou, China; ${ }^{2}$ Key Laboratory of Endocrine Gland Diseases of Zhejiang Province, Hangzhou, China; ${ }^{3}$ Department of Head and \\ Neck \& Thyroid Surgery, Zhejiang Provincial People's Hospital, People’s Hospital of Hangzhou Medical College, Hangzhou, China; ${ }^{4}$ Department \\ of Pharmacy, Institute of Cancer Research and Basic Medical Sciences of Chinese Academy of Sciences, Cancer Hospital of University of Chinese \\ Academy of Sciences, Zhejiang Cancer Hospital, Hangzhou, China; ${ }^{5}$ Academician Workstation, School of Stomatology, Changsha Medical \\ University, Changsha, China; ${ }^{6}$ Department of Surgery, Seoul National University College of Medicine, Seoul Metropolitan Government, Seoul \\ National University Boramae Medical Center, Transdisciplinary Department of Medicine \& Advanced Technology, Seoul National University \\ Hospital, Seoul, Korea \\ Contributions: (I) Conception and design: Y Liu, Y Zhang; (II) Administrative support: P Huang, M Ge, C Zheng; (III) Provision of study materials \\ or patients: Z Tan, J Wang; (IV) Collection and assembly of data: Y Hu; (V) Data analysis and interpretation: J Sun; (VI) Manuscript writing: All \\ authors; (VII) Final approval of manuscript: All authors. \\ Correspondence to: Chuanming Zheng. Department of Head and Neck \& Thyroid Surgery, Zhejiang Provincial People's Hospital, People's Hospital of \\ Hangzhou Medical College, 158\# Shangtang Road, Hangzhou 310010, China. Email: mingdoc@163.com.
}

Background Anaplastic thyroid carcinoma (ATC) is an extremely aggressive solid tumor with no effective treatment at present. Because of the rapid growth and aggressiveness, nearly all patients die within six months after developing ATC. Hence, more research regarding novel therapeutic targets for ATC is urgently needed.

Methods: Single-cell RNA sequencing data and microarray data of ATC were retrieved from the Gene Expression Omnibus (GEO) database. Cell clustering was performed using the Seurat package. Then, differential expression and functional enrichment analyses were performed. Gene set enrichment analysis (GSEA) was further used to investigate the functional enrichment of lysyl oxidase (LOX) and bone morphogenetic protein-1 (BMP1). The expression levels of LOX and BMP1 were measured using quantitative real-time PCR and Western blot. LOX and BMP1 were knocked down using si-RNAs. Cell proliferation was evaluated by the CCK- 8 and clone formation assays. Cell migration and invasion were assessed by the wound healing assay and Transwell assay, respectively.

Results: LOX was upregulated at the single-cell level, as well as in ATC tissues and cell lines. LOX knockdown significantly inhibited ATC cell proliferation. Furthermore, the migration and invasion of ATC cells were remarkably inhibited after LOX inhibition. In addition, BMP1 regulated LOX expression in 8505C cells, while BMP1 overexpression restored the LOX activity blocked by the LOX inhibitor BAPN. BMP1 could also induce the cell proliferation and metastasis of ATC.

Conclusions: LOX/BMP1 mediates the malignant progression of ATC, highlighting the potential application of LOX/BMP1 in the treatment of ATC. This study provides new insights for efficient therapeutic agents based on the LOX/BMP1 axis.

Keywords: Lysyl oxidase (LOX); bone morphogenetic protein-1 (BMP1); anaplastic thyroid carcinoma (ATC)

Submitted Nov 09, 2021. Accepted for publication Jan 14, 2022.

doi: 10.21037 /gs-21-908

View this article at: https://dx.doi.org/10.21037/gs-21-908 


\section{Introduction}

Thyroid carcinomas are one of the most common endocrine cancers, including papillary thyroid carcinoma (PTC), follicular carcinoma, anaplastic thyroid carcinoma (ATC), and medullary carcinoma (1). Although most thyroid cancers are localized and are usually associated with good survival, population-based studies have documented a significant increase in the incidence of thyroid cancer worldwide across all pathological types over the past decades (2). ATC was arise from the follicular cells of the thyroid gland and compared to other thyroid cancers, ATC cells do not retain any of the biological features of normal follicular cells, such as thyroglobulin synthesis, iodine uptake, and thyroid stimulating hormone dependence (3). Characterized by aggressive local disease, rapid growth, high rates of metastasis, and fatal clinical outcomes, the median survival of patients with ATC was less than three to six months (4). Therefore, more studies regarding valid and practical therapeutic targets for ATC are urgently needed.

Previous studies have revealed several biological factors that may have predictive or prognostic relevance for ATC by means of immunohistochemistry and bioinformatics. The lysyl oxidase (LOX) family is included among the identified biomarkers. LOX is an enzyme that catalyzes the cross-linking of elastin and collagen in the extracellular matrix, thus controlling the tensile strength of tissues $(5,6)$. Furthermore, LOX family proteins have been shown in multiple studies to drive the processes of carcinoma progression, including gene expression regulation, cell growth, invasion, and metastasis $(7,8)$. LOX has thus been suggested as a promising strategy to prevent metastasis and invasion of ATC. Whether LOX is involved in ATC progression is yet to be established, and the specificity and effects of LOX expression in malignant and non-malignant cells in ATC are unclear. This current study aimed to investigate the functional role of intracellular LOX in ATC.

LOX is a forty-eight $\mathrm{kd}$ protein and $\mathrm{N}$-glycosylation and signal peptide cleavage are essential for LOX protein activation. After that, the $50 \mathrm{kd}$ proenzyme is secreted and converted into a mature, active $30 \mathrm{kd}$ form. In addition, the catalytic activity of LOX can be specifically and irreversibly inhibited by beta-aminopropionitrile (BAPN) (9). Procollagen C proteinase/bone morphogenetic protein-1 (BMP1) was originally identified as a secreted metalloprotease of the astacin metalloproteinase family, which plays a role in LOX proteolytic processing $(10,11)$.
By inducing the initial cleavage and release of some complexes, such as TGF- $\beta$, BMP1 has been regarded to be involved in tumor progression (12). For example, BMP1 overexpression reflects poor prognosis in clear cell renal cell carcinoma (13), whereas the role of BMP1 in thyroid carcinomas, especially in ATC, has never been reported.

In the present study, we accessed both single-cell sequencing data and microarray data from public databases and analyzed them by several computational biology methods. We also examined the expression of LOX in ATC tissues, PTC tissues, and cell lines. Then, we investigated the regulatory effect of LOX and BMP1 on the proliferation, migration, and invasion of ATC cells. Further validation of the interaction between LOX/BMP1 was conducted to determine whether LOX/BMP1 could be identified as a novel target of ATC.

We present the following article in accordance with the MDAR reporting checklist (available at https:// gs.amegroups.com/article/view/10.21037/gs-21-908/rc).

\section{Methods}

\section{Data sources}

Single-cell sequencing ATC data (GSE148673, number of samples: 5) and normal data (GSE134355, number of samples: 2) were acquired from the Gene Expression Omnibus (GEO) database (www.ncbi.nlm.nih.gov/ geo). Four microarray datasets (GSE29265, GSE33630, GSE65144, and GSE76039) associated with ATC were also extracted from the GEO database. Information about cell types was acquired from annotations of the downloaded data.

\section{Data processing}

$\mathrm{R}$ software (version: 4.0.4) was used for all the analyses in the study.

\section{Data quality control and data merging}

The single-cell sequencing data was read and filtered with Scrublet (14). The cells were screened out according to $200<$ nFeature_RNA $<8,000$, nCount_RNA $>10$, and mitochondria-expressed genes $<20 \%$. The batch effect from microarray data was removed by the "limma" package (function: removeBatchEffect()) while preserving differences between groups. 
Table 1 Clinical and pathological characteristics of patients

\begin{tabular}{|c|c|c|c|}
\hline Patient No. & Histology & Age (years) & Sex \\
\hline 1 & PTC & 65 & Male \\
\hline 2 & PTC & 68 & Female \\
\hline 3 & PTC & 58 & Male \\
\hline 4 & PTC & 54 & Male \\
\hline 5 & PTC & 54 & Male \\
\hline 6 & PTC & 67 & Female \\
\hline 7 & PTC & 43 & Female \\
\hline 8 & PTC & 42 & Male \\
\hline 9 & PTC & 58 & Male \\
\hline 10 & PTC & 58 & Female \\
\hline 11 & PTC & 58 & Male \\
\hline 12 & PTC & 59 & Female \\
\hline 13 & PTC & 22 & Female \\
\hline 14 & PTC & 66 & Male \\
\hline 15 & PTC & 21 & Female \\
\hline 16 & PTC & 25 & Female \\
\hline 17 & PTC & 47 & Female \\
\hline 18 & PTC & 28 & Female \\
\hline 19 & PTC & 52 & Male \\
\hline 20 & PTC & 45 & Female \\
\hline 21 & PTC & 62 & Female \\
\hline 22 & PTC & 56 & Female \\
\hline 23 & PTC & 50 & Female \\
\hline 24 & PTC & 54 & Female \\
\hline 25 & PTC & 48 & Male \\
\hline 26 & PTC & 48 & Male \\
\hline 27 & PTC & 55 & Male \\
\hline 28 & PTC & 53 & Male \\
\hline 29 & ATC & 75 & Male \\
\hline 30 & ATC & 54 & Male \\
\hline 31 & ATC & 47 & Male \\
\hline 32 & ATC & 72 & Female \\
\hline 33 & ATC & 77 & Male \\
\hline
\end{tabular}

PTC, papillary thyroid cancer; ATC, anaplastic thyroid cancer.

\section{Single-cell copy number variation analysis and cell type} annotation

We applied CopyKAT to analyze copy number variations of the single-cell sequencing data (15). Aneuploid copy number profiles which have diploid genomes are common $(88 \%)$ in tumors but are not found in normal cell types, by means of identifying of aneuploid copy number profiles, It is effective to separate normal cells from tumor cells (16). Aneuploidy cells were defined as malignant ATC cells. In regard of the batch effects in different samples, $\mathrm{R}$ package Harmony was used to integrate these samples to eliminate the batch effects. After standardizing and scaling the data, principal component analysis (PCA) was conducted for the purpose of dimensionality reduction (17). UMAP was applied to cluster and visualize data which was then identified and annotated with the SingleR package.

\section{LOX expression analyses}

The expression of LOX in single-cell sequencing samples was obtained and visualized by the Seurat package. We defined LOX-positive groups as LOX $>0$ while the others were negative groups. We screened the top 20 LOX-related marker genes from LOX-positive groups by performing differential gene expression analysis. The expression of these LOX-related marker genes in single-cell sequencing and microarray data were visualized by a heatmap.

\section{Gene set enrichment analysis (GSEA)}

GSEA (http://software.broadinstitute.org/gsea/index.jsp) was used to assess LOX- or BMP1-related pathways and molecular mechanisms in ATC samples (18). Enriched gene sets with $\mathrm{P}$ values of $<0.05$ were considered statistically significant.

\section{Expression analyses based on ATC, PTC, and normal tissues}

Samples of ATC, PTC, and normal tissues were obtained from ATC patients and PTC patients who were performed surgery in Zhejiang Cancer Hospital, Clinical and pathological characteristics of the patients were shown in Table 1. This study was approved by the Ethics Committee of Zhejiang Cancer Hospital (No. 2020QT259) and performed according to the Declaration of Helsinki (as 
Table 2 The primers of the genes

\begin{tabular}{ll}
\hline Gene symbol & Forward/reverse primer \\
\hline LOX & Forward: 5'-TCCAGGACTGAGAAAGGGGAA-3' \\
& Reverse: 5'-GGGTGGGATTCAGACCCTTC-3' \\
BMP1 & Forward: 5'-CACACCTGTGTCACCTTCCT-3' \\
& Reverse: 5'-CCGAGCCGCAAGGTC-3' \\
GAPDH & Forward: 5'-GTCATCCATGACAACTTTGG-3' \\
& Reverse: 5'-GAGCTTGACAAAGTGGTCGT-3' \\
\hline
\end{tabular}

revised in 2013) and Good Clinical Practice guidelines. Patients provided written informed consent for their tissue to be used in this study. Total RNA was extracted using TRIzol $^{\circledR}$ reagent (Cat No.15596-018, Life Technologies, Carlsbad, CA, USA) following the manufacturer's instructions and checked to inspect RNA integrity. Qualified total RNA was further purified by the RNeasy mini kit (Cat No.74106, QIAGEN, GmBH, Hilden, Germany) and labeled. Each slide was hybridized with $600 \mathrm{ng} \mathrm{Cy} 3-$ labeled cRNA using the Gene Expression Hybridization Kit (Cat No. 5188-5242, Agilent Technologies, Santa Clara, CA, USA), then slides were scanned with default settings. The original data from the gene microarray was adjusted using the limma package (19) and screened for differentially expressed genes (DEGs) using $2^{\mid \log 2 \text { (fold change)! }}>1$ with $\mathrm{P}<0.05$.

\section{Cell culture}

The PTC cell line BCPAP was obtained from Professor Haixia Guan (Department of Endocrinology and Metabolism, The First Affiliated Hospital of China Medical University, Shenyang, China), and the ATC cell line $8505 \mathrm{C}$ was purchased from the Shanghai Cell Bank of the Chinese Academy of Sciences (Shanghai, China). The thyroid epithelial cell line Nthy-ori3-1 was bought from the Shanghai Institute of Cell Biology, Chinese Academy of Sciences (Shanghai, China). Nthy-ori3-1 cells and BCPAP cells were cultured in RPMI 1640 and $8505 \mathrm{C}$ cells were cultured in MEM. All the medium was added with streptomycin $(100 \mathrm{U} / \mathrm{mL})$, penicillin $(100 \mathrm{U} / \mathrm{mL})$ and $10 \%$ fetal bovine serum (FBS). Cultures were incubated at $37{ }^{\circ} \mathrm{C}$ in a humidified atmosphere of $5 \% \mathrm{CO}_{2}$.

\section{Cell transfection}

Cells were plated into 6-well plates one day before transfection. Transfection was performed with si-LOX, si-BMP1, and the corresponding scramble control (siNC) (Applied Biological Materials Inc., Vancouver, BC, CANADA) using Lipofectamine ${ }^{\circledR} 3000$ (Invitrogen; Thermo Fisher Scientific, Inc., Waltham, MA, USA), according to the manufacturer's protocol. After transfection for $6 \mathrm{~h}$, the culture medium was removed and replaced with fresh DMEM containing 10\% FBS.

\section{Total RNA extraction and real-time PCR quantification}

Total RNA was isolated using the TRIzol $^{\circledR}$ reagent (Invitrogen; Thermo Fisher Scientific, Inc., Waltham, MA, USA), and quantified by absorbance at OD $260 \mathrm{~nm}$. The purity of the RNA was assessed by measuring the ratio of OD $260 \mathrm{~nm} / \mathrm{OD} 280 \mathrm{~nm}$. The total RNA was reversetranscribed into cDNA using the PrimeScript RT Reagent kit (Takara Biotechnology Co., Ltd., Dalian, China). Table 2 is the primers for related genes. All the PCR reactions were carried out using SYBRs Premix Dimer-Eraser ${ }^{\mathrm{TM}}$ (Takara Biotechnology Co., Ltd., Dalian, China). The real-time PCR assays were conducted by LightCycler 480 (Roche Diagnostics) and the mRNA expression was normalized by GAPDH.

\section{Western blot assay}

Cells were washed with pre-cooled phosphate-buffered saline (PBS) on ice and then boiled in SDS sample buffer. Proteins samples were electrophoresed on polyacrylamide gel electrophoresis gels to resolve, and then transferred to polyvinylidene fluoride membranes. After blocking with skim milk, the membranes were incubated with primary antibodies against the following proteins: LOX, BMP1, and $\beta$-actin (Proteintech, Rosemont, IL, USA). Membranes were then blotted with horseradish peroxidase (HRP)conjugated secondary antibodies (Jackson Immuno Research Laboratories, West Grove, PA, USA). The images of the gels were scanned using the Bio-Rad Gel Doc XR+ system (Bio-Rad, Hercules, CA, USA).

\section{Co-immunoprecipitation (co-IP) assays}

PureProteome Protein G Magnetic Beads (Millipore Corp., Billerica, MA) were washed 3 times with PBS contain $0.01 \%$ Tween 20 and centrifuged at 2,500 $\times \mathrm{g}$ for 5 minutes. Then the beads were suspended in PBS with $2 \mu \mathrm{g}$ of the specific antibodies for 2 hours at $4{ }^{\circ} \mathrm{C}$. After incubation, 
the beads were centrifuged at 2,500 $\times \mathrm{g}$ and removed the supernatant. After washing them with PBS, the beads were then resuspended in protein extract in immunoprecipitation buffer and PI for $500 \mu \mathrm{L}$ total volume, the samples were rotated overnight at $4{ }^{\circ} \mathrm{C}$. The beads were recovered by centrifugation at 2,500 $\times \mathrm{g}$ for 5 minutes and washed 3 times in PBS before solubilizing in $20 \mu \mathrm{L}$ RIPA buffer. Loading dye ( $5 \mu \mathrm{L}$ of a $5 \times$ solution) was added and the beads were resuspended prior to preparation for SDS-PAGE.

\section{Cell counting kit-8 (CCK-8) assay}

Cells were seeded into 96 -well plates at $5 \times 10^{3}$ cells/well. The CCK- 8 assay was performed to detect cell proliferation ability at $72 \mathrm{~h}$ following inoculation. In brief, $10 \mu \mathrm{L}$ of CCK-8 dye (Beyotime Institute of Biotechnology, Shanghai, China) was added into each well and cells were incubated for $1 \mathrm{~h}$ at $37{ }^{\circ} \mathrm{C}$ under $5 \% \mathrm{CO}_{2}$. Finally, we used microplate reader (Bio-Rad Laboratories, Hercules, CA, USA) to obtain the absorbance of each well at $450 \mathrm{~nm}$.

\section{Clone formation assay}

From each group, approximately $1 \times 10^{3}$ cells were plated in each well of a 6-well culture plate. Cells were incubated at $37^{\circ} \mathrm{C}$ for 2 weeks with culture media being replaced every 3 days. Then, the cells were washed 3 times with PBS and stained with $0.5 \%$ crystal violet. Photomicrographs were taken at a magnification of $10 \times$ for all groups.

\section{Transwell assay}

The cells invasive ability was determined by the Transwell assay. Cells were cultured in $100 \mu \mathrm{L}$ serum-free medium after transfection for 48 hours. Cells were plated into the upper chamber containing a Transwell pre-coated Matrigel membrane filter and $8 \mu \mathrm{m}$ pore inserted in the culture plates. The cells remaining on the upper membrane were removed with cotton swabs after $48 \mathrm{~h}$ incubation. As for the cells which had invaded through the membrane, they were fixed in $4 \%$ paraformaldehyde and stained with $0.1 \%$ crystal violet for $20 \mathrm{~min}$ at $4{ }^{\circ} \mathrm{C}$. Counts were finally obtained from 5 random fields at $10 \times$ magnification.

\section{Scratch wound healing assay}

The migratory ability of cells was analyzed using a wound healing assay. Briefly, cells were plated on 6-well plates and scraped by a pipette tip to generate uniform wounds. The initial gap length at $0 \mathrm{~h}$ and the residual gap length at $24 \mathrm{~h}$ after wounding were observed and photographed at a magnification of $10 \times$ for each group.

\section{LOX activity assay}

ATC cells conditioned media $(50 \mu \mathrm{L})$ was added to $150 \mu \mathrm{L}$ of reaction mixture containing $10.6 \mathrm{mM}$ cadaverine (SigmaAldrich), $1 \mathrm{M}$ urea (Sigma-Aldrich), $0.2 \mathrm{U} / \mathrm{mL}$ HRP (Sigma-Aldrich), and $20 \mu \mathrm{M}$ amplex red (Molecular Probes, Invitrogen) in the 96-well clear-bottom black plate (Perkin Elmer, Waltham, MA). Plates were incubated at $37^{\circ} \mathrm{C}$ for $1 \mathrm{~h}$. Finally, microplate reader (Bio-Rad Laboratories, Hercules, CA, USA) was used to read the absorbance of each well at $544 \mathrm{~nm}$ excitation and $590 \mathrm{~nm}$ emission.

\section{Correlation analysis}

The matrix of gene expression data [GSE29265 (n=9)] associated with ATC was extracted from the GEO database (http://www.ncbi.nlm.nih.gov/geo/; species: homo). The Pearson method was used to determine the correlation coefficient. The R4.0.1 Ggstatsplot package was used to visualize the correlation analysis results.

\section{Immunofluorescence}

Cells were plated onto coverslips and fixed in cold methanol for 10 minutes. Then the cells were blocked with $5 \%$ FBS and incubated with the primary antibodies and secondary antibodies. The sections were counterstained with 4,6-diamino-2-phenylindole and observed under a fluorescence microscope. Photomicrographs were taken for all groups.

\section{Statistical analysis}

All the experiments were performed 3 times independently, 
A
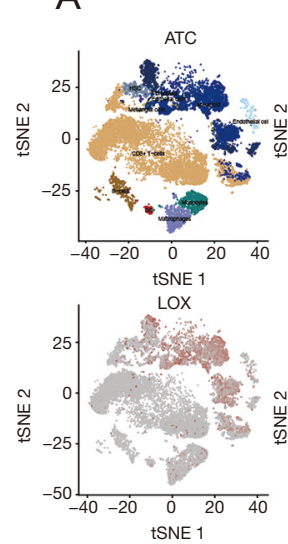
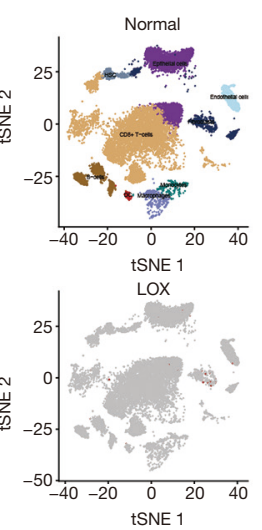

B

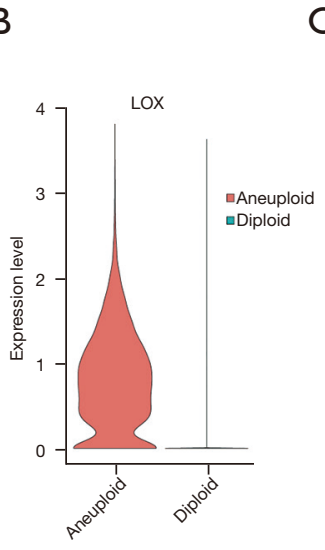

C

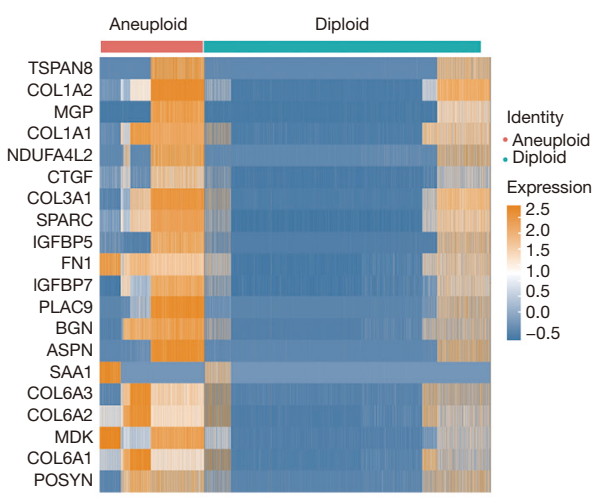

Figure 1 LOX expression in ATC cells delineated by single-cell sequencing analysis. (A) tSNE of single-cell profiling with each cell colorcoded for sample type and associated cell type (up). Expression of LOX for the cell types defined above each panel (down). (B) Violin plots show the expression levels of LOX in malignant ATC cells and normal cells. (C) Heatmap representing the upregulated and downregulated top 20 LOX-related genes in aneuploid and diploid clusters. t-SNE, t-distributed stochastic neighbour embedding; LOX, Lysyl oxidase; ATC, anaplastic thyroid carcinoma.

and the experiments data were analyzed by means of GraphPad Prism software (La Jolla, CA, USA). Results are presented as mean $\pm S D$. Differences between groups were tested using paired and paired Student's $t$-tests. $\mathrm{P}<0.05$ was considered statistically significant.

\section{Results}

\section{Single-cell profiling and clustering of ATC cells}

First, single-cell sequencing analysis was performed to analyze LOX expression. ATC and normal cells were both grouped into 11 clusters. LOX expression in malignant ATC cells was significantly higher than that of normal cells $(\mathrm{P}<0.05)$ (Figure 1A,1B). By leveraging single-cell melanoma expression data, we identified gene profiles that were significantly co-expressed with $L O X$ in different cell types, and the heatmap shows the top 20 marker genes in aneuploid and diploid subsets (Figure 1C). Overall, LOX showed abnormally high expression at the single-cell level.

\section{LOX plays an important role in ATC and is associated with proliferation and migration}

We used microarray data from the GEO dataset to verify the expression profiles of the above-mentioned LOXrelated genes in Figure 1, and 14 of the top 20 genes were overexpressed in ATC (Figure 2A). Consistent with single- cell sequencing analysis, LOX expression in the ATC group $(\mathrm{n}=52)$ was markedly higher than in the normal group $(\mathrm{n}=78)$ from microarray analysis (median 7.48 vs. 4.20, $\mathrm{P}<0.05$ ) (Figure $2 B$ ), which proved that LOX are malignant gene expressed in ATC. GSEA showed that the high LOX expression group was mainly enriched in Gene Ontology (GO) biological processes related to cell population proliferation and cell migration (Figure $2 C$ ).

\section{LOX is significantly upregulated in ATC tissues}

We first examined the expression of LOX in ATC, PTC, and normal tissues, and a significantly higher LOX level was observed in ATC tissues (Figure $3 A, 3 B)(\mathrm{P}<0.01)$. Subsequently, total RNA was extracted from the thyroid epithelial cell line Nthy-ori3-1, the PTC cell line BCPAP, and the ATC cell line $8505 \mathrm{C}$. RT-qPCR analysis revealed that $L O X$ expression was significantly increased in $8505 \mathrm{C}$ cells compared to BCPAP and Nthy-ori3-1 cells (Figure 3C, $\mathrm{P}<0.05)$. These results indicated that LOX was upregulated in ATC.

\section{Knockdown of LOX suppresses the proliferation, migration, and invasion of ATC cells}

LOX was significantly knocked down in $8505 \mathrm{C}$ cells after transfection with si-RNA (Figure $4 A, \mathrm{P}<0.01$ ). LOX activity was also decreased in si-LOX $8505 \mathrm{C}$ cells 

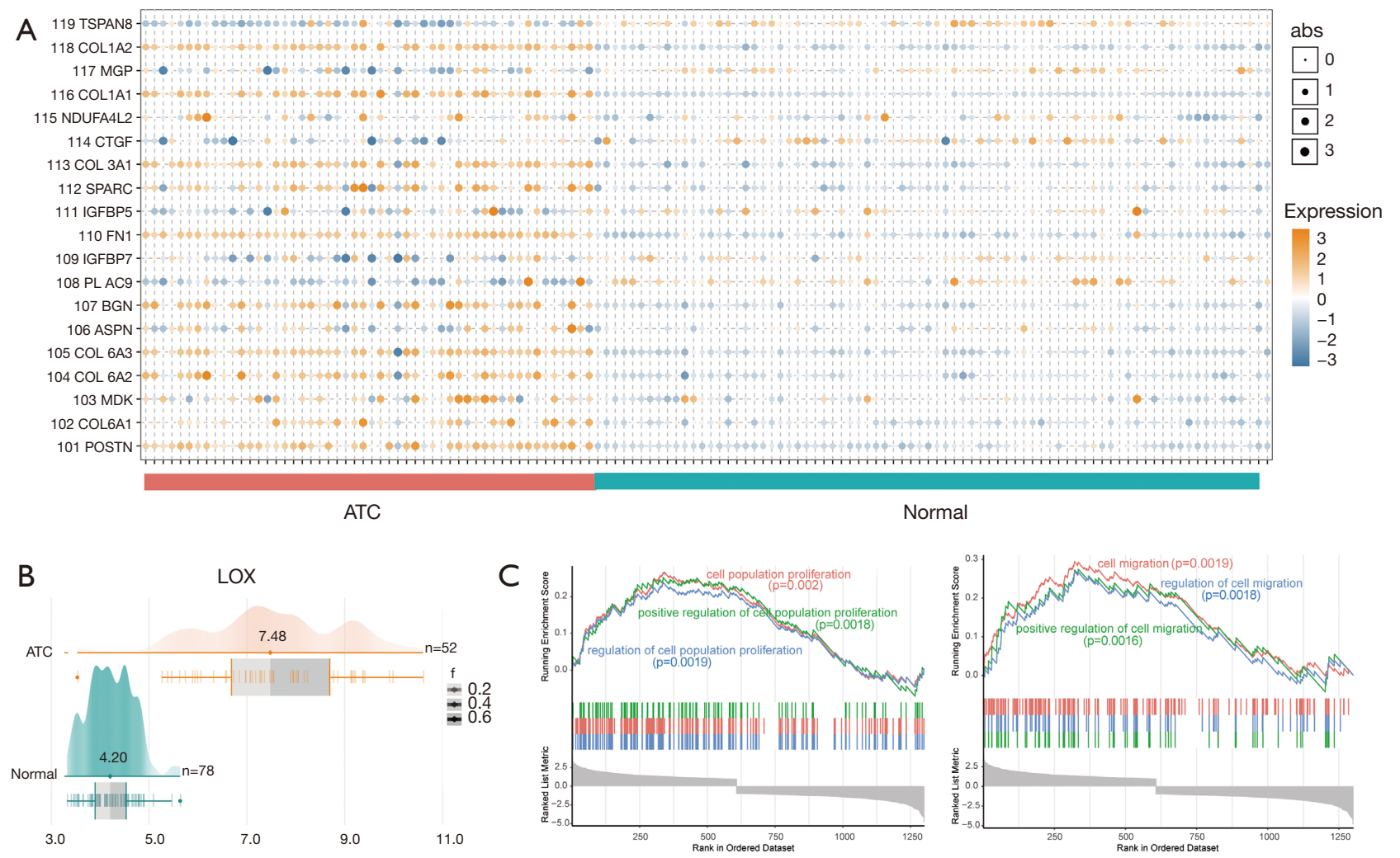

Figure 2 The expression of LOX was upregulated in ATC tissues and cell lines. (A) Heatmaps representing the upregulated and downregulated top 20 LOX-related genes in ATC and normal subsets using microarray analysis. (B) The expression level of LOX in the ATC and normal groups (7.48 vs. 4.20). (C) GSEA showing high-LOX groups are associated with cell proliferation- and migration-related GO pathways. LOX, Lysyl oxidase; ATC, anaplastic thyroid carcinoma; GSEA, gene set enrichment analysis; GO, Gene Ontology.

(Figure $4 B, \mathrm{P}<0.001$ ). Both growth curves and colony formation efficiency showed that LOX downregulation impeded the proliferation of $8505 \mathrm{C}$ cells compared with cells transfected with control (Figure $4 C, 4 D, \mathrm{P}<0.01$ ). In addition, the migratory and invasive abilities of $8505 \mathrm{C}$ cells were examined using the Transwell migration and scratch wound healing assays. The numbers of invaded (Figure 4E) and migrated (Figure 4F) 8505C cells were much lower in the in si-LOX group than in the control group. Similar results were observed in $8505 \mathrm{C}$ cells treated with the LOX inhibitor BAPN (Figure 4G,4H). All these results illustrated the effects of LOX in the malignant progression of ATC.

\section{The effect of BMP1 in the proliferation and metastasis of ATC cells}

A previous study revealed that LOX can be activated by
BMP1 in other tumors. In this study, BMP1 expression in the ATC group was markedly higher than in the normal group as determined by microarray analysis (median 6.02 vs. 5.39, $\mathrm{P}<0.05$ ) (Figure $5 A$ ). We conducted GSEA to determine the molecular function of BMP1 in ATC, and the high-BMP1 group was also enriched in cell proliferationand migration-related GO pathways (Figure 5B). To confirm whether BMP1 plays a role in promoting the proliferation and metastasis of $8505 \mathrm{C}$ cells, we knocked down BMP1 by si-RNA. The CCK- 8 and clone formation assays both demonstrated that BMP1 knockdown could reduce ATC cell viability (Figure 5C,5D). The migratory and invasive abilities of $8505 \mathrm{C}$ cells were also impaired by BMP1 knockdown (Figure 5E, 5F). Moreover, si-BMP1 could enhance the inhibition effect of BAPN. Taken together, BMP1 protein exerted a promotive effect on ATC malignant progression, and therefore may be regarded as a 

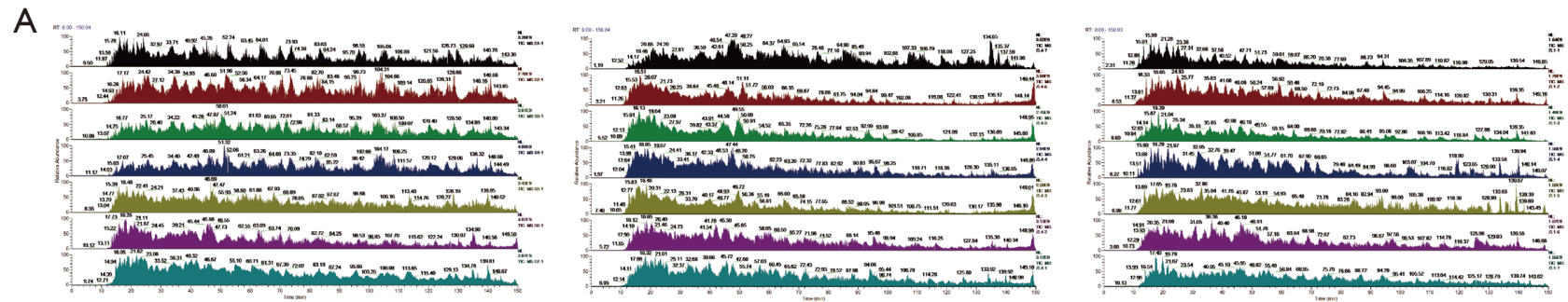

B

\begin{tabular}{ccc}
\multicolumn{3}{c}{ Signal intensity of LOX } \\
\hline Normal & PTC & ATC \\
\hline n.d & n.d & $1.87 \times 10^{5}$ \\
n.d & n.d & $3.63 \times 10^{5}$ \\
n.d & n.d & $6.92 \times 10^{5}$ \\
n.d & n.d & $5.37 \times 10^{5}$ \\
n.d & n.d & $3.42 \times 10^{5}$ \\
n.d & n.d & $7.65 \times 10^{5}$ \\
n.d & n.d & $5.30 \times 10^{5}$ \\
\hline
\end{tabular}

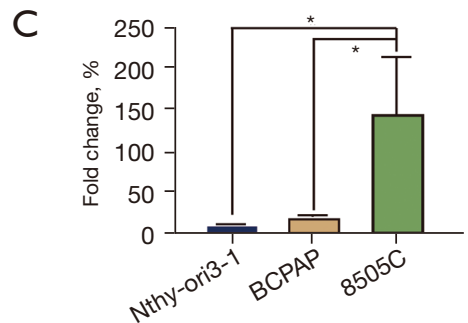

Figure 3 The expression of LOX was upregulated in ATC tissues and cell lines. (A) The protein expression level of LOX in ATC, PTC, and normal tissues was measured using protein mass spectrometry analysis. (B) The mRNA level of LOX in ATC, PTC, and normal tissues was determined by quantitative real-time PCR. (C) The mRNA level of $L O X$ in Nthy-ori3-1, BCPAP, and 8505C cells was detected using quantitative real-time PCR. *, P<0.05. LOX, Lysyl oxidase; ATC, anaplastic thyroid carcinoma; PTC, papillary thyroid carcinoma; mRNA, messenger RNA.

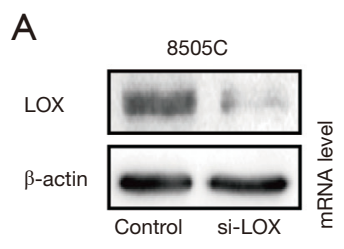

E

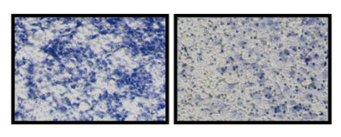

Control

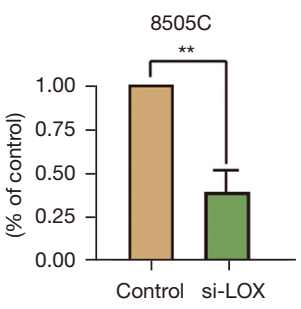

$\mathrm{F}$

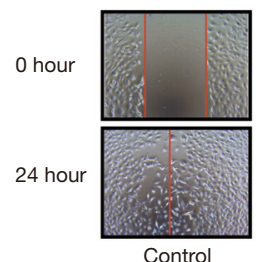

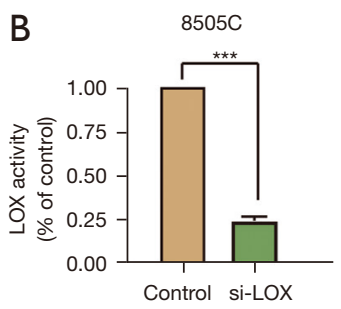

G

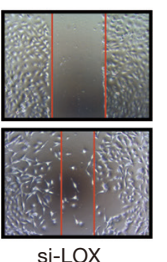

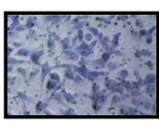

Control
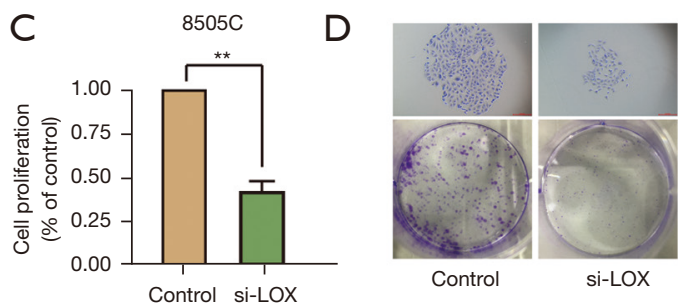

$\mathrm{H}$
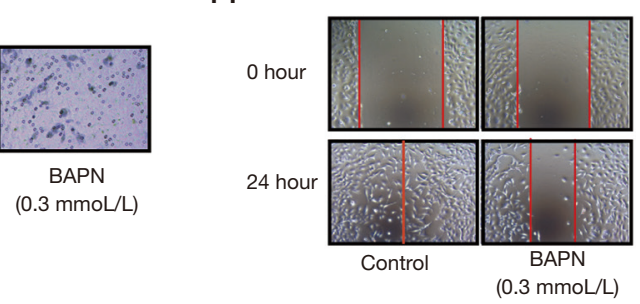

Figure 4 LOX knockdown inhibited the proliferation, migration, and invasion of ATC cells. (A) The expression of LOX was knocked down using si-RNAs in $8505 \mathrm{C}$ cells. (B) The activity of LOX was downregulated after knocking down LOX in 8505C cells. (C) The proliferation of $8505 \mathrm{C}$ cells after LOX knockdown was assessed using the cell counting kit-8 assay. (D) The proliferative ability of $8505 \mathrm{C}$ cells after LOX silencing was evaluated using the clone formation assay (crystal violet staining at 10× magnification). (E,G) Cell migration of $8505 \mathrm{C}$ cells was evaluated by the wound healing assay (at 10x magnification). (F,H) Cell invasion of $8505 \mathrm{C}$ cells was evaluated by the Transwell assay (crystal violet staining at $10 \times$ magnification). ${ }^{* *}, \mathrm{P}<0.01$; ${ }^{* * *}, \mathrm{P}<0.001$. LOX, Lysyl oxidase; si-RNAs, short interfering RNAs. 

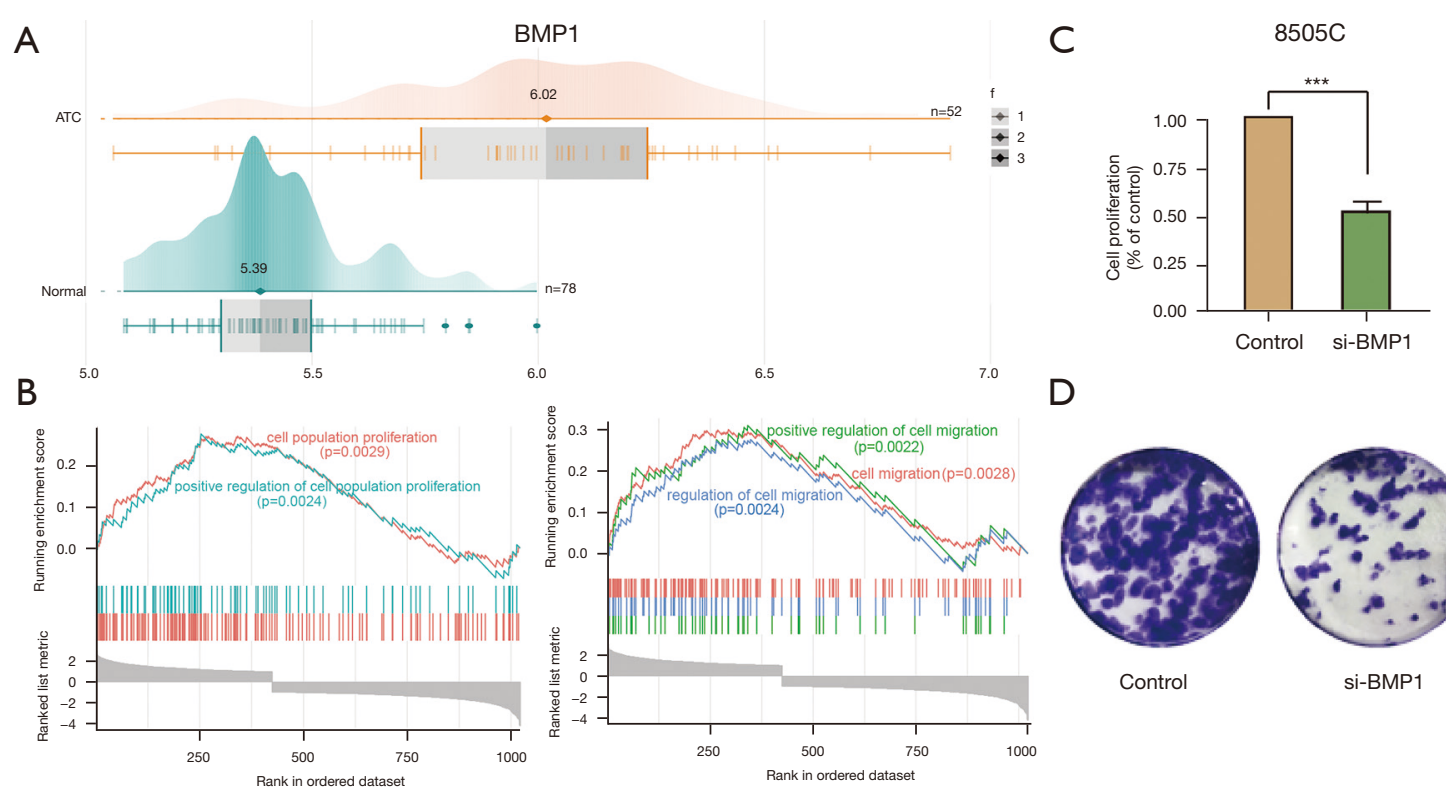

$\mathrm{D}$
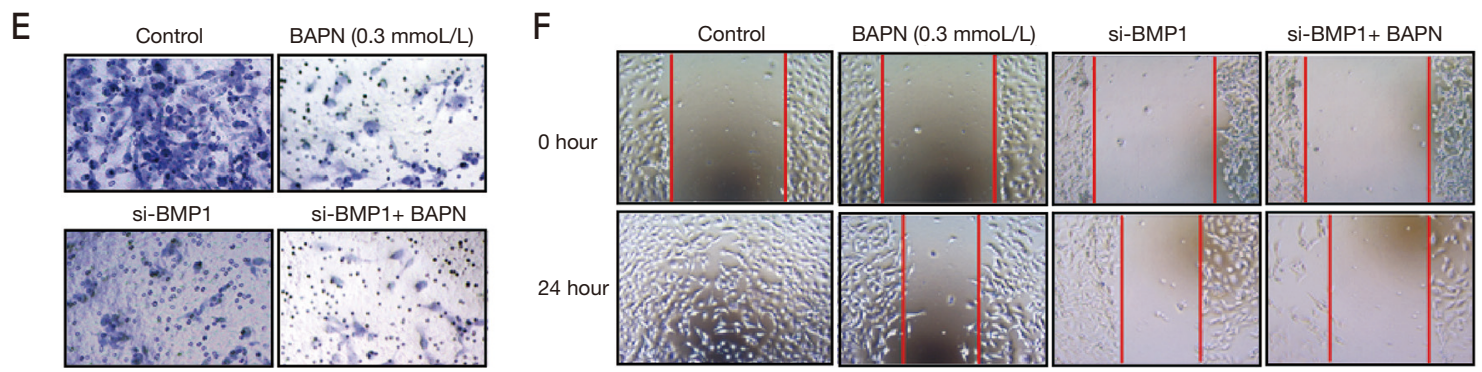

Figure 5 The effect of BMP1 on the proliferation and metastasis of ATC cells. (A) The expression level of BMP1 in the ATC and normal groups (6.02 vs. 5.39). (B) GSEA showing that the high-BMP1 group was associated with cell proliferation- and migration-related GO pathways. (C,D) The proliferative ability of $8505 \mathrm{C}$ cells was evaluated by the cell counting kit- 8 assay (C) and clone formation assay (D) (crystal violet staining at $10 \times$ magnification). (E) Cell invasion of $8505 \mathrm{C}$ cells was evaluated by the Transwell assay (crystal violet staining at $10 \times$ magnification). (F) Cell migration of $8505 \mathrm{C}$ cells was evaluated by the wound healing assay (at $10 \times$ magnification). ***, $\mathrm{P}<0.001$. BMP-1, bone morphogenetic protein-1; ATC, anaplastic thyroid carcinoma; GSEA, gene set enrichment analysis; GO, Gene Ontology; PTC, papillary thyroid carcinoma.

new therapeutic target of ATC.

\section{Association between LOX and BMP1 in ATC cells}

The correlation between BMP1 and LOX, as determined by the Person method based on the GEO database (Figure $6 A$ ), was $\mathrm{R}=0.52$, which indicates a significant positive correlation. In order to investigate the association between LOX and BMP1 in ATC cells, we conducted an immunofluorescence assay to analyze the co-localization of BMP1 and LOX proteins in $8505 \mathrm{C}$ cells (Figure 6B). Co-IP revealed that BMP1 might bind with LOX in $8505 \mathrm{C}$ cells (Figure 6C).

\section{BMP1 could mediate the activity and expression of $L O X$ in ATC cells}

In order to further investigate the effect of BMP1 on the function of LOX in ATC, we knocked down BMP1 by si-RNA (Figure 7A). Compared with the control group, RT-PCR and Western blot assays revealed that LOX was significantly downregulated in si-BMP1 $8505 \mathrm{C}$ cells (Figure $7 B, \mathrm{P}<0.01$ ). Furthermore, the activity of LOX was also decreased when BMP1 was knocked down (Figure $7 C$, $\mathrm{P}<0.05)$. When BMP1 was overexpressed, the activity of LOX inhibited by BAPN could be rescued, as shown in Figure $7 D$. These results showed that BMP1 could mediate 

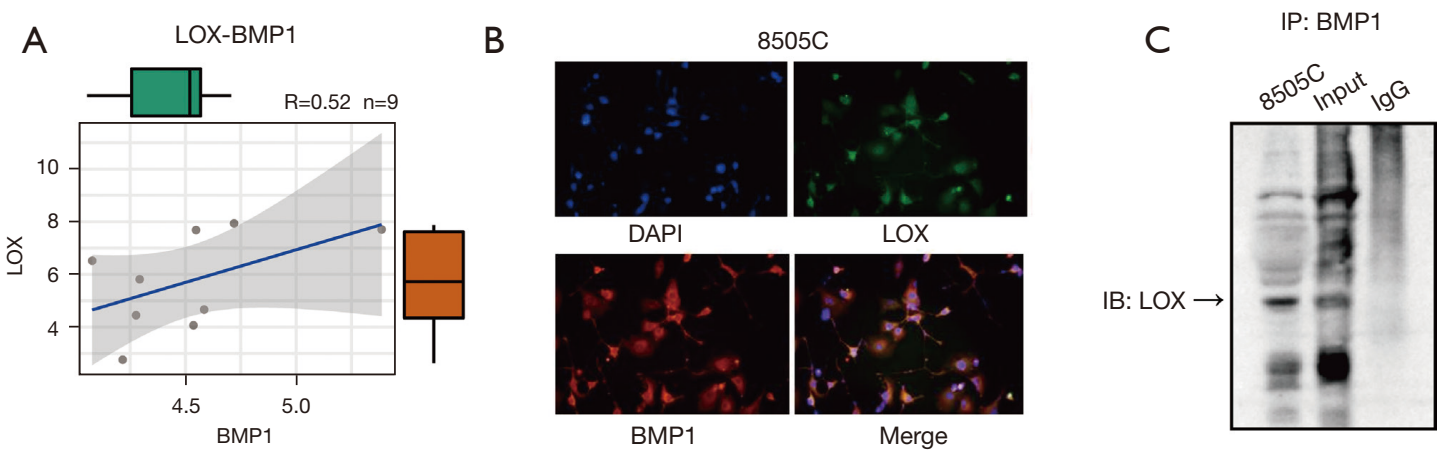

Figure 6 Association between LOX and BMP1 in ATC cells. (A) The correlation between BMP1 and LOX using the Pearson method based on the GEO database. (B) The co-localization of BMP1 and LOX proteins in 8505C cells was shown by immunofluorescence (at 10x magnification). (C) The interaction between BMP1 and LOX was detected using co-IP. BMP-1, bone morphogenetic protein-1; LOX, Lysyl oxidase; ATC, anaplastic thyroid carcinoma; co-IP, co-immunoprecipitation.

A

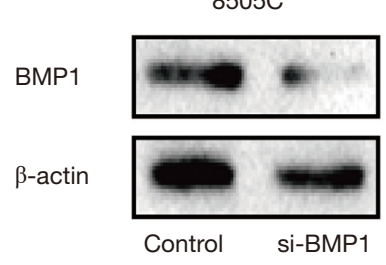

C

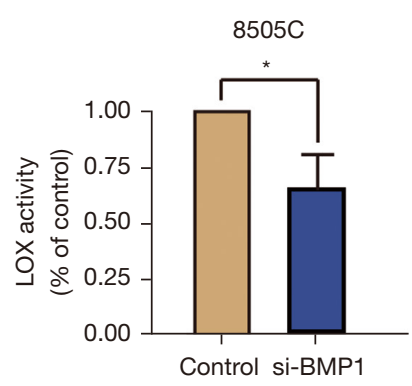

B

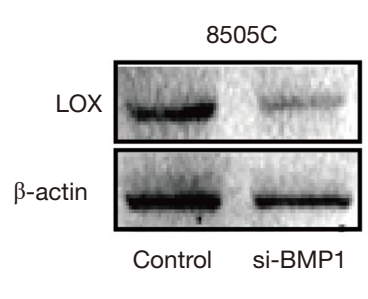

$8505 \mathrm{C}$

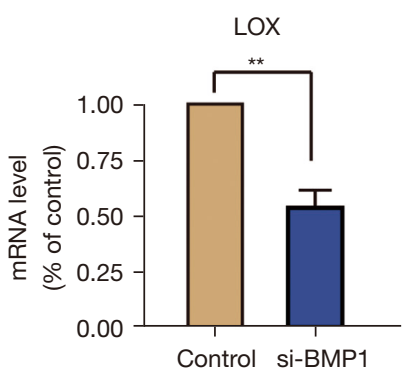

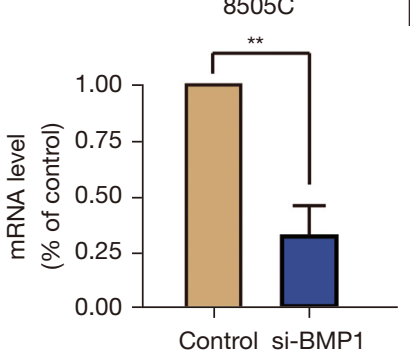

D

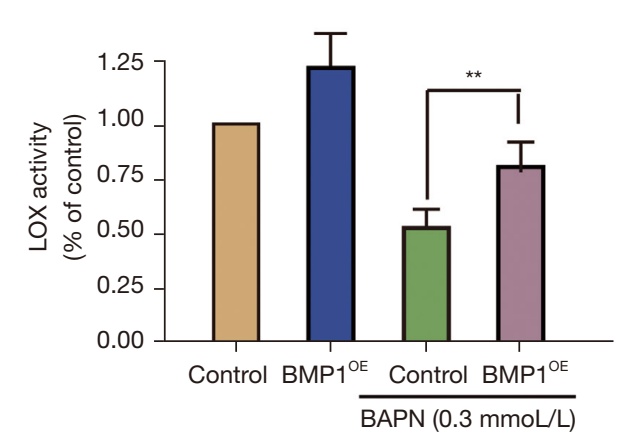

Figure 7 BMP1 could mediate the activity and expression of LOX in ATC cells. (A) The expression of BMP1 was knocked down using si-RNAs in $8505 \mathrm{C}$ cells. (B) The expression level of LOX in 8505C cells after si-BMP1 transfection was measured by Western blot and quantitative real-time PCR. (C) The activity of LOX was downregulated after knocking down BMP1 in 8505C cells. (D) The activity of LOX was determined after BMP1 overexpression and treatment with BAPN in $8505 \mathrm{C}$ cells. ${ }^{*}, \mathrm{P}<0.05 ;{ }^{* *}, \mathrm{P}<0.01$. BMP-1, bone morphogenetic protein-1; si-RNAs, short interfering RNAs; LOX, Lysyl oxidase; BAPN, beta-aminopropionitrile (LOX inhibitor).

the activity and expression of LOX in ATC cells.

\section{Discussion}

In this study, we found abnormal expression of LOX and
BMP1 in ATC from the single-cell and gene expression level up to cell lines or tumor tissue level.

LOX is a secreted amine oxidase that causes stiffening of the matrix and plays a crucial role in mediating tumor microenvironment, which enhances the invasive and 
metastatic properties of the tumor (20). Previous study showed that LOX was significantly associated with breast cancer bone metastasis. High expression of LOX in primary breast tumors leads to osteolytic lesion formation (5). Therefore, LOX can prompt more aggressive tumor behavior.

Using the retrieved single-cell sequencing data, the molecular signature of ATC tumor tissues was analyzed. In tumor cells, a high expression of LOX was found at the single-cell level, and a subset of LOX-related genes was identified. In support of this, we examined LOX expression in thyroid tumor tissues. LOX expression was undetectable in normal tissue, thyroid epithelial cell line, and PTC cell line, whereas LOX had high abnormal expression in ATC tissues samples and the ATC cell line (8505C). Furthermore, functional analysis demonstrated that knocking down LOX with siRNA or inhibiting LOX with BAPN led to a significant reduction in the migration and invasion of $8505 \mathrm{C}$ cells. BAPN was obtained originally from sweet peas, Lathyrus odoratus $\mathrm{L}$ and was the first chemical compound described as LOX potent irreversible inhibitor. BAPN has shown anticancer properties in several different cancer types. Such findings are in agreement with a previous study that LOX exacerbated the malignant and invasive properties of cancer.

BMP1 was reported that was responsible for processing LOX into the active form (21), and an association between LOX activation and BMP1 has been previously reported in other cancers (22). Similar results were confirmed in this study by knocking down BMP1 in ATC. To further understand the interaction between BMP1 and LOX expression in ATC, we used co-IP and immunofluorescence co-localization analysis. Our results identified relevant protein-protein interactions of LOX and BMP1. Importantly, GSEA was performed and demonstrated that BMP1 was related to cell proliferation and migration. For validation, the in vitro assays demonstrated that siBMP1 could inhibit ATC cell proliferation and invasion. Moreover, BMP1 overexpression restored the activation of LOX repressed by BAPN in $8505 \mathrm{C}$ cells. Although there are no current agents that are known to target LOX/BMP1, our results suggest that LOX/BMP1 depletion or LOX inhibition could be an effective treatment strategy in ATC.

Functional enrichment analysis of the LOX-related genes from ATC cells may help to define the specific roles of LOX expression in malignant tumor development and progression. However, we do not have further information on the involvement of LOX in influencing PTC cell proliferation and migration through these genes. In future work, we will further explore the relationship between them.

However, our study had limitations. Our study provides evidence that LOX and BMP1 promote the proliferation and migration of ATC cells. But the analysis was only conducted in vitro study, further in vivo experiments of ATC need to be explored in future studies.

Overall, our results confirmed that LOX plays an important role in ATC malignant progression. BMP1 can activate LOX and promote the proliferation and migration of ATC cells. The inhibition of LOX and BMP1 was suggested as a promising therapeutic strategy for oncological diseases, including ATC. In the upcoming years, we expect to obtain more detailed information on the research of LOX and BMP1. The combination of LOX enzyme inhibitors with standard anticancer treatments is another approach that should be further explored in future studies. Our data will be contributed to the progress of therapeutic development against ATC.

\section{Acknowledgments}

The authors appreciate the academic support from the AME Thyroid Cancer Collaborative Group.

Funding: This work was supported by the National Natural Science Foundation of China (grant number 8217131437); Projects of International Cooperation and Exchanges NSFC (grant number 8211101233); Zhejiang Provincial Natural Science Foundation of China, Zhejiang Province, People's Republic of China (grant numbers LYQ20H310001 and LYY21H310011); the Medical and Health Research Program of Zhejiang Province (grant numbers 2018KY297, 2019KY047, 2021KY055 2021KY040, 2022RC008, and 2022KY069); Chinese Medicine Research Program of Zhejiang Province (grant number 2021ZZ001); Medical and Health Research Program of Zhejiang (grant number WKJZJ-2115); "Pioneer" and "Leading Goose" R\&D Program of Zhejiang (grant number 2022C03116); Zhejiang Provincial Program for the Cultivation of New Heath Talents (to Yiwen Zhang); "10000 Talents Plan” of Zhejiang Province (to Ping Huang, grant number 2020R52029); "10000 Talents Plan" of Zhejiang Province (to Minghua Ge, grant number 2017R52028) and Key Research and Development Program of Zhejiang Province (grant number 2021C03081).

\section{Footnote}

Reporting Checklist: The authors have completed the MDAR 
reporting checklist. Available at https://gs.amegroups.com/ article/view/10.21037/gs-21-908/rc

Data Sharing Statement: Available at https://gs.amegroups. com/article/view/10.21037/gs-21-908/dss

Conflicts of Interest: All authors have completed the ICMJE uniform disclosure form (available at https://gs.amegroups. com/article/view/10.21037/gs-21-908/coif). YL reports funding from the Zhejiang Provincial Natural Science Foundation of China, Zhejiang Province, China (grant number LYQ20H310001); the Medical and Health Research Program of Zhejiang Province (grant numbers 2018KY297, 2022RC008). YZ reports funding from the Medical and Health Research Program of Zhejiang Province (grant numbers 2021KY040, 2022KY069); Chinese Medicine Research Program of Zhejiang Province (grant number 2021ZZ001); Zhejiang Provincial Program for the Cultivation of New Heath Talents (to YZ). JW reports funding from the Medical and Health Research Program of Zhejiang Province (grant number 2021KY055). YH reports funding from the Zhejiang Provincial Natural Science Foundation of China, Zhejiang Province, China (grant number LYY21H310011). JS reports funding from the Medical and Health Research Program of Zhejiang Province (grant number 2019KY047). PH reports funding from the National Natural Science Foundation of China (grant number 8217131437); Projects of International Cooperation and Exchanges NSFC (grant number 8211101233); Medical and Health Research Program of Zhejiang grant number WKJ-ZJ-2115); "Pioneer" and "Leading Goose" R\&D Program of Zhejiang (grant number 2022C03116); "10000 Talents Plan" of Zhejiang Province (to PH, grant number 2020R52029). MG reports funding from " 10000 Talents Plan" of Zhejiang Province (to MG, grant number 2017R52028) and Key Research and Development Program of Zhejiang Province (grant number 2021C03081). The other authors have no conflicts of interest to declare.

Ethical Statement: The authors are accountable for all aspects of the work in ensuring that questions related to the accuracy or integrity of any part of the work are appropriately investigated and resolved. This study was approved by the Ethics Committee of Zhejiang Cancer Hospital (No. 2020QT259), and performed according to the Declaration of Helsinki (as revised in 2013) and Good Clinical Practice guidelines. Patients provided written informed consent for their tissue to be used in this study.

Open Access Statement: This is an Open Access article distributed in accordance with the Creative Commons Attribution-NonCommercial-NoDerivs 4.0 International License (CC BY-NC-ND 4.0), which permits the noncommercial replication and distribution of the article with the strict proviso that no changes or edits are made and the original work is properly cited (including links to both the formal publication through the relevant DOI and the license). See: https://creativecommons.org/licenses/by-nc-nd/4.0/.

\section{References}

1. Seib CD, Sosa JA. Evolving Understanding of the Epidemiology of Thyroid Cancer. Endocrinol Metab Clin North Am 2019;48:23-35.

2. Molinaro E, Romei C, Biagini A, et al. Anaplastic thyroid carcinoma: from clinicopathology to genetics and advanced therapies. Nat Rev Endocrinol 2017;13:644-60.

3. Nath MC, Erickson LA. Aggressive Variants of Papillary Thyroid Carcinoma: Hobnail, Tall Cell, Columnar, and Solid. Adv Anat Pathol 2018;25:172-9.

4. Kebebew E, Greenspan FS, Clark OH, et al. Anaplastic thyroid carcinoma. Treatment outcome and prognostic factors. Cancer 2005;103:1330-5.

5. Cox TR, Erler JT. Lysyl oxidase in colorectal cancer. Am J Physiol Gastrointest Liver Physiol 2013;305:G659-66.

6. Trackman PC. Functional importance of lysyl oxidase family propeptide regions. J Cell Commun Signal 2018;12:45-53.

7. Mitra S, Deshmukh A, Sachdeva R, et al. Oxidized lowdensity lipoprotein and atherosclerosis implications in antioxidant therapy. Am J Med Sci 2011;342:135-42.

8. De Donato M, Petrillo M, Martinelli E, et al. Uncovering the role of nuclear Lysyl oxidase (LOX) in advanced high grade serous ovarian cancer. Gynecol Oncol 2017;146:170-8.

9. Smith-Mungo LI, Kagan HM. Lysyl oxidase: properties, regulation and multiple functions in biology. Matrix Biol 1998;16:387-98.

10. Hays TT, Ma B, Zhou N, et al. Vascular smooth muscle cells direct extracellular dysregulation in aortic stiffening of hypertensive rats. Aging Cell 2018;17:e12748.

11. Vadon-Le Goff S, Hulmes DJ, Moali C. BMP-1/tolloidlike proteinases synchronize matrix assembly with growth factor activation to promote morphogenesis and tissue remodeling. Matrix Biol 2015;44-46:14-23. 
12. Hyytiäinen M, Penttinen C, Keski-Oja J. Latent TGFbeta binding proteins: extracellular matrix association and roles in TGF-beta activation. Crit Rev Clin Lab Sci 2004;41:233-64.

13. Xiao W, Wang X, Wang T, et al. Overexpression of BMP1 reflects poor prognosis in clear cell renal cell carcinoma. Cancer Gene Ther 2020;27:330-40.

14. Wolock SL, Lopez R, Klein AM. Scrublet: Computational Identification of Cell Doublets in Single-Cell Transcriptomic Data. Cell Syst 2019;8:281-291.e9.

15. Gao R, Bai S, Henderson YC, et al. Delineating copy number and clonal substructure in human tumors from single-cell transcriptomes. Nat Biotechnol 2021;39:599-608.

16. Taylor AM, Shih J, Ha G, et al. Genomic and Functional Approaches to Understanding Cancer Aneuploidy. Cancer Cell 2018;33:676-689.e3.

17. Butler A, Hoffman P, Smibert P, et al. Integrating singlecell transcriptomic data across different conditions, technologies, and species. Nat Biotechnol 2018;36:411-20.

Cite this article as: Liu Y, Zhang Y, Tan Z, Wang J, Hu Y, Sun J, Bao M, Huang P, Ge M, Chai YJ, Zheng C. Lysyl oxidase promotes anaplastic thyroid carcinoma cell proliferation and metastasis mediated via BMP1. Gland Surg 2022;11(1):245257. doi: $10.21037 / \mathrm{gs}-21-908$
18. Subramanian A, Kuehn H, Gould J, et al. GSEA-P: a desktop application for Gene Set Enrichment Analysis. Bioinformatics 2007;23:3251-3.

19. Ritchie ME, Phipson B, Wu D, et al. limma powers differential expression analyses for RNA-sequencing and microarray studies. Nucleic Acids Res 2015;43:e47.

20. Miller BW, Morton JP, Pinese M, et al. Targeting the LOX/hypoxia axis reverses many of the features that make pancreatic cancer deadly: inhibition of LOX abrogates metastasis and enhances drug efficacy. EMBO Mol Med 2015;7:1063-76.

21. Maruhashi T, Kii I, Saito M, et al. Interaction between periostin and BMP-1 promotes proteolytic activation of lysyl oxidase. J Biol Chem 2010;285:13294-303.

22. da Silva R, Uno M, Marie SK, et al. LOX expression and functional analysis in astrocytomas and impact of IDH1 mutation. PLoS One 2015;10:e0119781.

(English Language Editor: C. Betlazar-Maseh) 